\title{
Monte carlo simulations of Yttrium reaction rates in Quinta ura- nium target
}

M. Suchopár ${ }^{1,2, \star}$, V. Wagner ${ }^{1,2}$, O. Svoboda ${ }^{1}, J$. Vrzalová ${ }^{1,2,4}, P$. Chudoba ${ }^{1,3}, P$. Tichý ${ }^{1,2,4}$, A. Kugler ${ }^{1}$, J. Adam ${ }^{1,4}$, L. Závorka ${ }^{2,4}$, A. Baldin ${ }^{4,5}$, W. Furman ${ }^{4}, M$. Kadykov $^{4, \dagger}$, J. Khushvaktov ${ }^{4}$, A. Solnyshkin ${ }^{4}$, V. Tsoupko-Sitnikov ${ }^{4}, S$. Tyutyunnikov ${ }^{4}, M$. Bielewicz ${ }^{4,6}, S^{2}$ Kilim $^{6}$, E. StrugalskaGola $^{6}$, and M. Szuta ${ }^{6}$

${ }^{1}$ Nuclear Physics Institute of the ASCR PRI, Hlavní 130, 25068 Řež, Czech Republic

${ }^{2}$ Faculty of Nuclear Sciences and Physical Engineering, Czech Technical University in Prague, Břehová 7 , 11519 Prague 1, Czech Republic

${ }^{3}$ Faculty of Mathematics and Physics, Charles University in Prague, Ke Karlovu 3, 12116 Prague 2, Czech Republic

${ }^{4}$ Joint Institute for Nuclear Research, Joliot-Curie 6, 141980 Dubna, Russia

${ }^{5}$ Institute for Advanced Studies "OMEGA", Universitetskaya 19, 141980 Dubna, Russia

${ }^{6}$ National Centre for Nuclear Research, 05-400 Otwock-Świerk, Poland

\begin{abstract}
The international collaboration Energy and Transmutation of Radioactive Waste (E\&T RAW) performed intensive studies of several simple accelerator-driven system (ADS) setups consisting of lead, uranium and graphite which were irradiated by relativistic proton and deuteron beams in the past years at the Joint Institute for Nuclear Research (JINR) in Dubna, Russia. The most recent setup called Quinta, consisting of natural uranium target-blanket and lead shielding, was irradiated by deuteron beams in the energy range between 1 and $8 \mathrm{GeV}$ in three accelerator runs at JINR Nuclotron in 2011 and 2012 with yttrium samples among others inserted inside the setup to measure the neutron flux in various places. Suitable activation detectors serve as one of possible tools for monitoring of proton and deuteron beams and for measurements of neutron field distribution in ADS studies. Yttrium is one of such suitable materials for monitoring of high energy neutrons. Various threshold reactions can be observed in yttrium samples. The yields of isotopes produced in the samples were determined using the activation method. Monte Carlo simulations of the reaction rates leading to production of different isotopes were performed in the MCNPX transport code and compared with the experimental results obtained from the yttrium samples.
\end{abstract}

\section{Introduction}

Yttrium as an activation material has several advantages when investigating fast neutron fluxes produced in spallation reactions of relativistic light ions impacting on thick heavy metal targets used in

^e-mail: suchopar@ujf.cas.cz

$\dagger$ deceased 
ADS studies [1-4]. Yttrium in natural occurrence is monoisotopic with one stable isotope ${ }^{89} \mathrm{Y}$. Products of $(n, x n)$ threshold reactions on yttrium are easily identifiable. Half-lives of the products have suitable duration for gamma spectroscopy. Gamma transitions are intensive enough for detection and they are also well separated from each other. The yttrium activation detectors were irradiated during experiments with the Quinta uranium target [5, 6] at the Joint Institute for Nuclear Research (JINR) in Dubna, Russia. The deuteron beams with energies ranging from $1 \mathrm{GeV}$ up to $8 \mathrm{GeV}$ were produced by JINR Nuclotron synchrotron. Residual radionuclides in the samples were measured by the gamma spectrometry.

\section{Experimental method and simulation procedure}

The samples had a form of pills made of compressed yttrium powder with a diameter of $10 \mathrm{~mm}$, a thickness of $1.5 \mathrm{~mm}$, and a weight of $\sim 0.6-0.8 \mathrm{~g}$ [9]. The purity of used ${ }^{89} \mathrm{Y}$ was $99.99 \%$. These samples were placed on the front side of the detector plates in two positions at radial distances of 4 and $8 \mathrm{~cm}$ from the target axis ( 5 and $8 \mathrm{~cm}$ in case of the first detector plate with a hole for the beam entrance). The aluminium detector plates were located in the gaps between the setup sections at longitudinal distances of $0,12.9,26.2,39.1,52.4$, and $65.3 \mathrm{~cm}$ from the target beginning. Schema of the experimental setup and locations of yttrium activation detectors in the Quinta assembly are depicted in figure 1. Twelve yttrium samples in total were used in each irradiation of the Quinta setup in 2, 4 and $6 \mathrm{GeV}$ deuteron experiments carried out in March 2011 (without lead shielding) [7], 1 and $4 \mathrm{GeV}$ deuteron experiments in December 2011 (with lead shielding) [9], and 2, 4 and $8 \mathrm{GeV}$ deuteron experiments in December 2012 (again with lead shielding) [8]. Products of ${ }^{89} \mathrm{Y}(\mathrm{n}, 2 \mathrm{n}){ }^{88} \mathrm{Y},{ }^{89} \mathrm{Y}(\mathrm{n}, 3 \mathrm{n}){ }^{87} \mathrm{Y}$, ${ }^{89} \mathrm{Y}(\mathrm{n}, 4 \mathrm{n}){ }^{86} \mathrm{Y}$, and ${ }^{89} \mathrm{Y}(\mathrm{n}, 5 \mathrm{n}){ }^{85} \mathrm{Y}$ reactions with threshold energies 11.6, 21.1, 33.0, and $42.6 \mathrm{MeV}$ respectively were detected using gamma spectroscopy and their yields per gram of activation material per beam deuteron were determined. The acquired gamma spectra were analysed using the Deimos 32 spectroscopy software [10] with all necessary spectroscopic corrections applied [11]. The yield of each isotope was then calculated as a weighted average of at least two gamma lines.

The beam integral was determined using an aluminium foil located at sufficient distance from the setup (about $3 \mathrm{~m}$ ) so that the measurement was not affected by backscattered neutrons from the setup and reaction rates from the activation foils were normalized to this beam monitor. The beam integral was determined with around $10 \%$ accuracy due to the fit of the excitation function of the ${ }^{27} \mathrm{Al}(\mathrm{d}, 3 \mathrm{p} 2 \mathrm{n}){ }^{24} \mathrm{Na}$ monitoring reaction as only 3 values for this reaction cross section are present in the EXFOR database in the GeV energy range $[12,13]$. The beam position and profile were determined using the solid state nuclear track detectors (SSNTD) by means of the measurement of distribution of induced fission rates by the beam in natural lead samples [14, 15]. A fit of the experimental data by Gaussian distribution in the horizontal and vertical direction was used as input parameters for the particle source in the Monte Carlo simulations.

We simulated the isotope production in threshold ( $\mathrm{n}, \mathrm{xn})$ reactions in yttrium samples and compared them with the experimental results obtained by the group from the National Centre for Nuclear Research in Otwock-Świerk, Poland [7-9]. The Monte Carlo simulations of the yields of activation reactions in the samples were performed with the MCNPX code version 2.7.0 [16]. Standard cross section libraries ENDF/B-VII.1 [17] included in the MCNPX code package were used for the simulations coupled with the INCL intranuclear cascade and ABLA fission-evaporation models for the primary beam interaction and high energy particle transport. Microscopic cross sections of ${ }^{89} \mathrm{Y}(\mathrm{n}, \mathrm{xn})$ threshold reactions were calculated by the Talys code version 1.6 [18] with default parameters of the level density models. The simulated yields were calculated by folding of the reaction cross sections calculated by Talys (up to $200 \mathrm{MeV}$ ) and MCNPX (up to the beam energy) with neutron, proton and deuteron spectra computed by MCNPX. The contribution to the total reaction yield is produced 
mainly by neutrons, protons and deuterons (near the beam axis), contribution by other particles (such as charged pions) can be neglected being on the level of the simulation uncertainties.
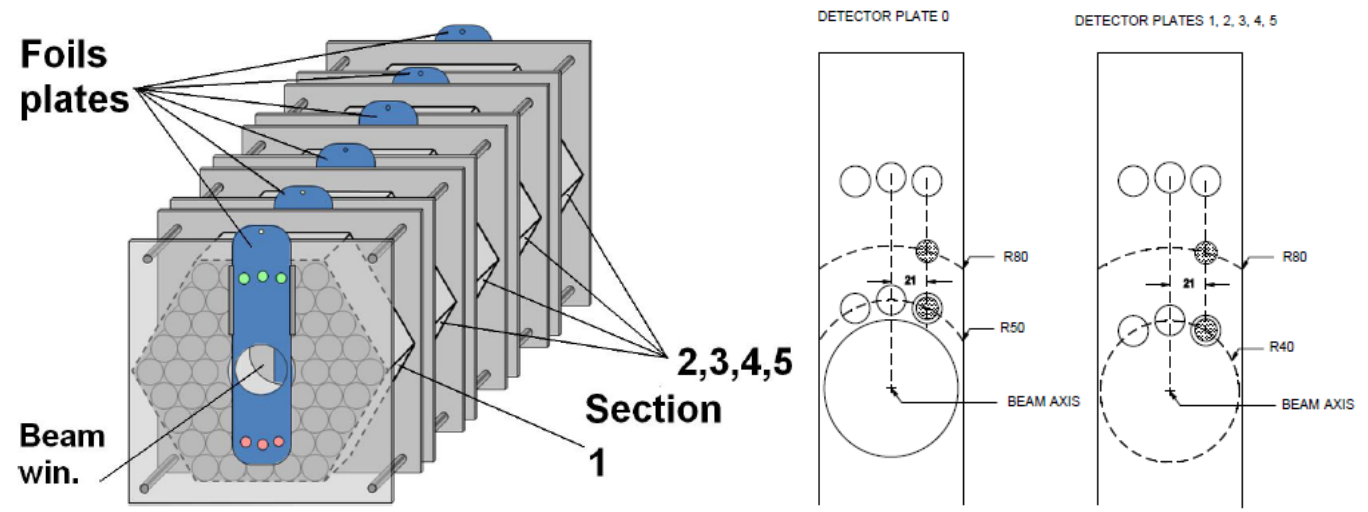

Figure 1. Schema of the Quinta setup (left). Locations of yttrium activation samples on the detector plates (right) [8]. Dimensions are in millimeters.

\section{Discussion of results}

After proper normalization of the data, we could compare the experimental spatial distributions of the reaction rates with our Monte Carlo calculations in the MCNPX code. The absolute and relative comparisons of experimental and simulated data in the axial direction in case of the three above mentioned $4 \mathrm{GeV}$ experiments are shown in figure 2-4. The ratio of experimental to simulated yields varies between 0.5 and 1.5 in most cases and confirms relatively good agreement between the experiment and simulation, although the simulation generally underestimates the experimental data. The only apparent discrepancy appears at the maximum of isotopes production which is located at about $26 \mathrm{~cm}$ from the target beginning where the simulation underestimates the experimental data by a factor of two for the position at $4 \mathrm{~cm}$ in the 2, 4 and $6 \mathrm{GeV}$ experiments performed without lead shielding but not in the rest of experiments where the Quinta setup was shielded with lead. This may be caused by a slight misplacement of the samples from their anticipated positions on the third plate due to the presence of other groups' samples rather than an incorrect beam displacement input in the simulation as the other foils near the beam axis are not influenced by this effect. Another discrepancy was observed in case of the $1 \mathrm{GeV}$ experiment but only for the front detector plate. It could be caused by inaccurate beam position determination on the front side of the target or inaccurate placement of the samples on the first detector plate. Therefore, it has to be emphasized that the results strongly depend on precise beam parameters determination and are very sensitive to accurate activation foils placement in the setup.

\section{Conclusion}

Measurements of fast neutron field in the Quinta assembly were performed by means of yttrium activation detectors irradiated with 1,2, 4, 6 and $8 \mathrm{GeV}$ deuteron beams from the Nuclotron accelerator at JINR, Dubna. Experimental data on $(\mathrm{n}, \mathrm{xn})$ reaction rate distributions provided by the group from 


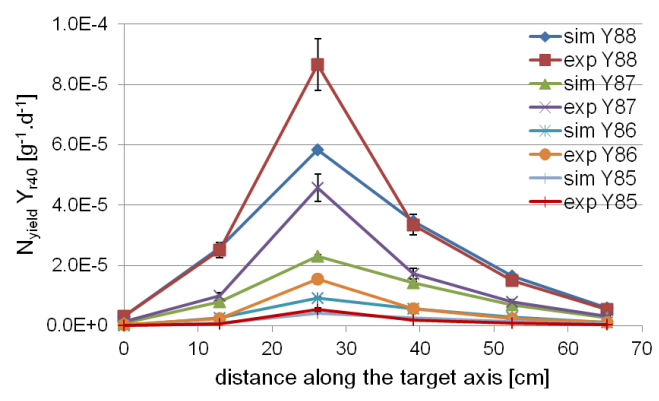

(a) radius $40 \mathrm{~mm}$

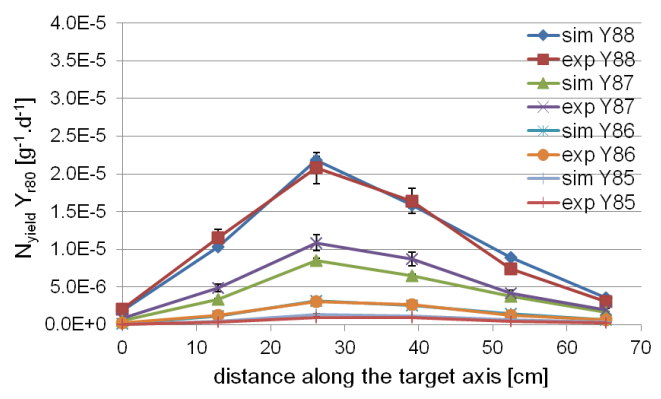

(c) radius $80 \mathrm{~mm}$

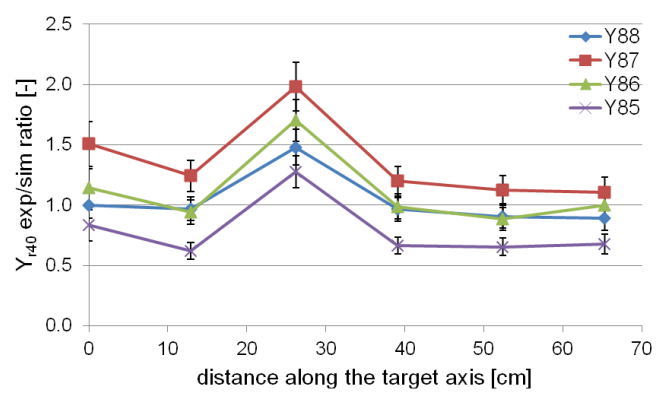

(b) radius $40 \mathrm{~mm}$, exp/sim ratio

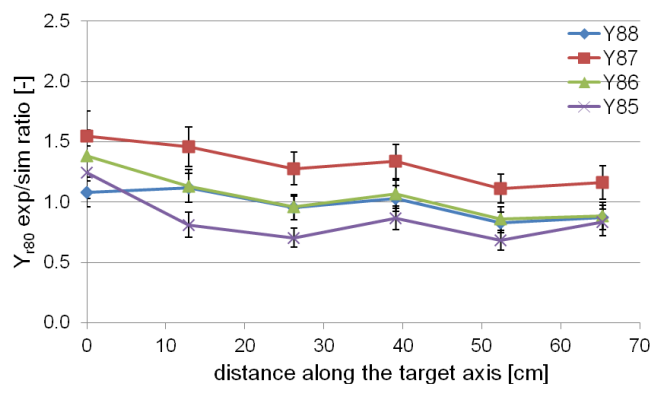

(d) radius $80 \mathrm{~mm}$, exp/sim ratio

Figure 2. Measured and calculated yields of observed isotopes in yttrium detectors in longitudinal direction at $40 \mathrm{~mm}$ (a) and $80 \mathrm{~mm}$ (c) above the target axis and relative comparison of experimental and simulated yields at $40 \mathrm{~mm}$ (b) and $80 \mathrm{~mm}$ (d) above the target axis, $4 \mathrm{GeV}$ experiment in March 2011.

the National Centre for Nuclear Research in Otwock-Świerk, Poland were compared with reaction rates calculated by the MCNPX code. The experimental spatial distribution of ${ }^{88} \mathrm{Y},{ }^{87} \mathrm{Y},{ }^{86} \mathrm{Y}$ and ${ }^{85} \mathrm{Y}$ production as well as the simulated one has a maximum at about $26 \mathrm{~cm}$ from the front of the target assembly as it was expected. The shape of reaction rate distribution is generally similar for all beam energies but differs in absolute value, which depends also on impacting beam position on the target. The ratio of experimental and simulated yields varies in the interval 0.5-2.0 in most cases and confirms relatively good agreement between the two, although the simulation generally underestimates the experimental data. Some discrepancy was observed at the maximum of isotopes production, where the simulation apparently underestimates the experimental data for the position at $4 \mathrm{~cm}$ in the 2,4 and 6 $\mathrm{GeV}$ experiments performed without lead shielding but not in other experiments with lead shielding in place.

\section{References}

[1] V. Wagner et al., PoS (Baldin ISHEPP XXI) 090 (2012)

[2] V. Wagner et al., Journal of Physics: Conference Series 366, 012047 (2012)

[3] P. Chudoba et al., PoS (Baldin ISHEPP XXII) 054 (2014)

[4] P. Chudoba et al., Physics Procedia 59, 114-118 (2014) 


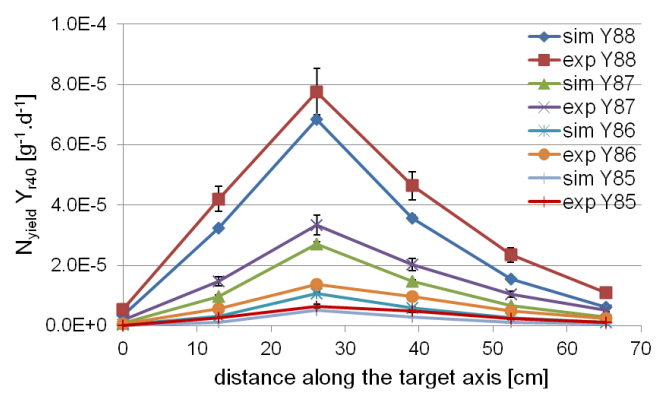

(a) radius $40 \mathrm{~mm}$

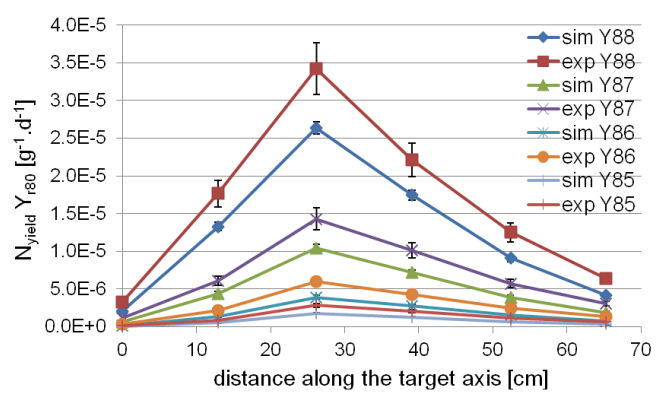

(c) radius $80 \mathrm{~mm}$

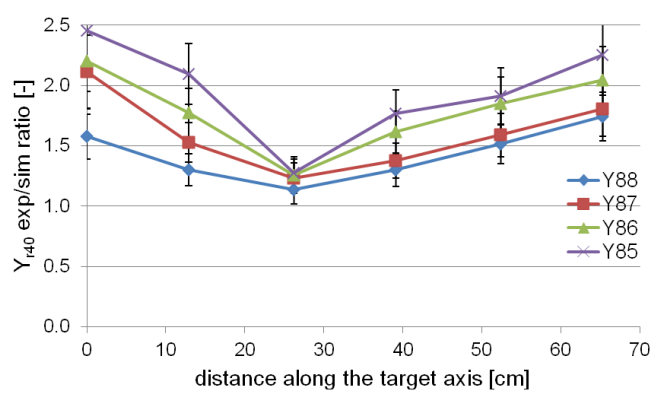

(b) radius $40 \mathrm{~mm}$, exp/sim ratio

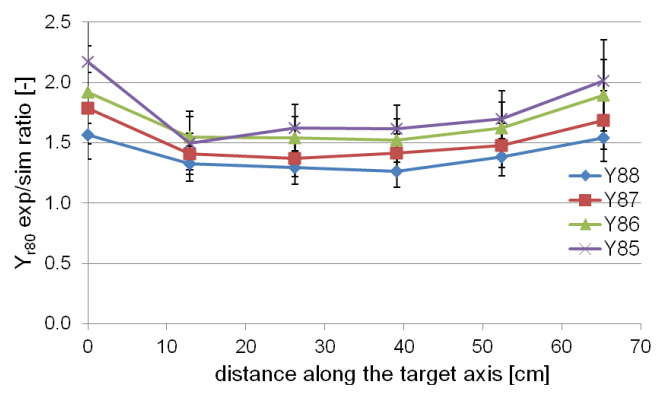

(d) radius $80 \mathrm{~mm}$, exp/sim ratio

Figure 3. Measured and calculated yields of observed isotopes in yttrium detectors in longitudinal direction at $40 \mathrm{~mm}$ (a) and $80 \mathrm{~mm}$ (c) above the target axis and relative comparison of experimental and simulated yields at $40 \mathrm{~mm}$ (b) and $80 \mathrm{~mm}$ (d) above the target axis, $4 \mathrm{GeV}$ experiment in December 2011.

[5] W. Furman et al., PoS (Baldin ISHEPP XXI) 086 (2012)

[6] M. Suchopár et al., PoS (Baldin ISHEPP XXI) 091 (2012)

[7] M.M. Bielewicz et al., PoS (Baldin ISHEPP XXI) 092 (2012)

[8] M.M. Bielewicz et al., PoS (Baldin ISHEPP XXII) 052 (2014)

[9] M. Szuta et al., PoS (Baldin ISHEPP XXII) 060 (2014)

[10] J. Frána, Journal of Radioanalytical and Nuclear Chemistry 257, 583-587 (2003)

[11] M. Majerle et al., JINR Preprint E11-2009-178 (Dubna, Russia, 2009)

[12] J. Banaigs et al., Nuclear Instruments and Methods in Physics Research 95, 307-311 (1971)

[13] P. Kozma and V.V. Yanovski, Czech Journal of Physics 40, 393 (1990)

[14] S.R. Hashemi-Nezhad et al., Nuclear Instruments and Methods in Physics Research A 664, 154$160(2012)$

[15] V. Bukhal et al., PoS (Baldin ISHEPP XXII) 053 (2014)

[16] D.B. Pelowitz, editor, MCNPX User's Manual, Version 2.7.0, LANL report LA-CP-11-00438 (Los Alamos, USA, 2011)

[17] M.B. Chadwick et al., Nuclear Data Sheets 112, 2887-2996 (2011)

[18] A.J. Koning et al., TALYS-1.6, A nuclear reaction program, User manual (NRG Petten, The Netherlands, 2013) 


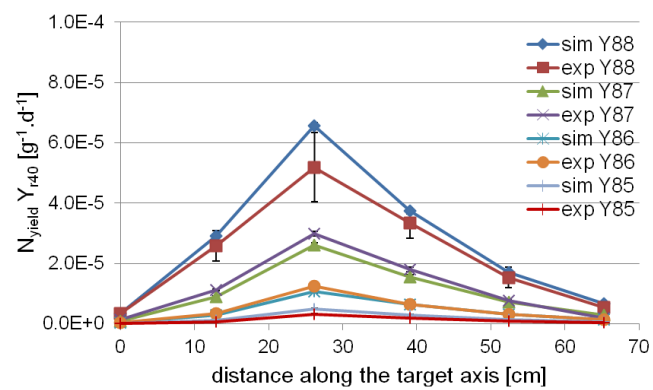

(a) radius $40 \mathrm{~mm}$

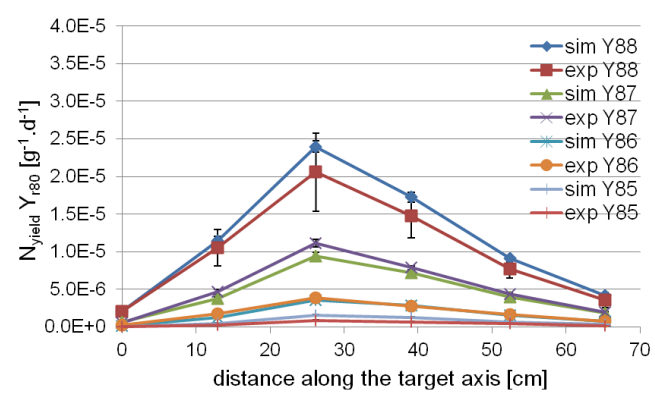

(c) radius $80 \mathrm{~mm}$

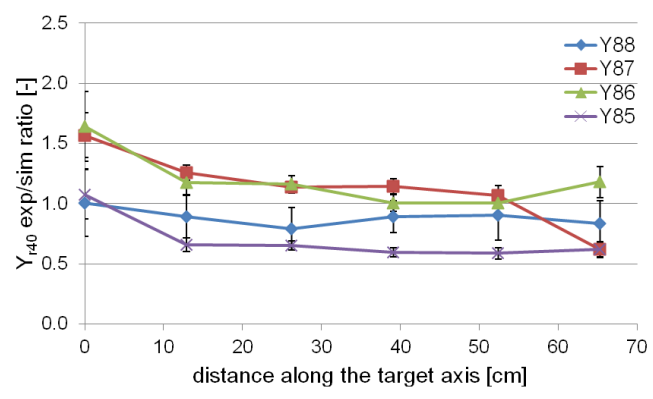

(b) radius $40 \mathrm{~mm}$, exp/sim ratio

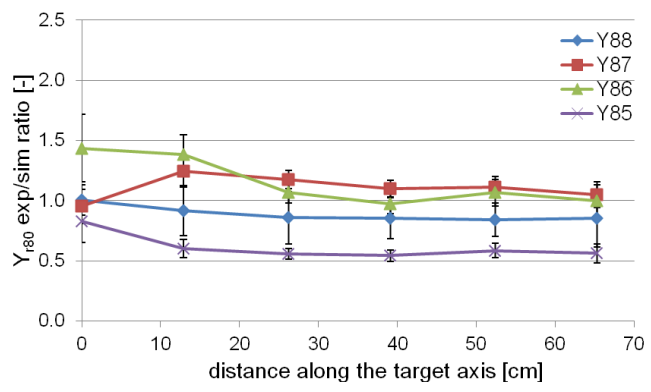

(d) radius $80 \mathrm{~mm}$, exp/sim ratio

Figure 4. Measured and calculated yields of observed isotopes in yttrium detectors in longitudinal direction at $40 \mathrm{~mm}$ (a) and $80 \mathrm{~mm}$ (c) above the target axis and relative comparison of experimental and simulated yields at $40 \mathrm{~mm}$ (b) and $80 \mathrm{~mm}$ (d) above the target axis, $4 \mathrm{GeV}$ experiment in December 2012. 